\title{
X-ray and optical monitoring of the December 2017 outburst of the Be/X-ray binary AX J0049.4-7323
}

\author{
L. Ducci ${ }^{1,2}$, C. Malacaria ${ }^{3,4}$, P. Romano ${ }^{5}$, L. Ji ${ }^{1}$, E. Bozzo ${ }^{2}$, I. Saathoff ${ }^{1}$, A. Santangelo ${ }^{1}$, and A. Udalski ${ }^{6}$ \\ ${ }^{1}$ Institut für Astronomie und Astrophysik, Kepler Center for Astro and Particle Physics, Eberhard Karls Universität, Sand 1, \\ 72076 Tübingen, Germany \\ e-mail: ducci@astro.uni-tuebingen.de \\ 2 ISDC Data Center for Astrophysics, Université de Genève, 16 chemin d'Écogia 1290 Versoix, Switzerland \\ 3 NASA Marshall Space Flight Center, NSSTC, 320 Sparkman Drive, Huntsville, AL 35805, USA \\ ${ }^{4}$ Universities Space Research Association, NSSTC, 320 Sparkman Drive, Huntsville, AL 35805, USA \\ 5 INAF - Osservatorio Astronomico di Brera, via Bianchi 46, 23807 Merate, LC, Italy \\ 6 Warsaw University Observatory, Al. Ujazdowskie 4, 00-478 Warszawa, Poland
}

Received 15 October 2018 / Accepted 12 November 2018

\begin{abstract}
AX J0049.4-7323 (SXP 756) is a Be/X-ray binary that shows an unusual and poorly understood optical variability that consists of periodic and bright optical outbursts, simultaneous with X-ray outbursts, characterised by a highly asymmetric profile. The periodicity of the outbursts is thought to correspond to the orbital period of the neutron star. To understand the peculiar behaviour shown by this source, we performed the first multi-wavelength monitoring campaign during the periastron passage of December 2017. The monitoring lasted for about 37 days and consisted of X-ray, near-ultraviolet, and optical data from the Neil Gehrels Swift Observatory, the optical $I$ band from the OGLE survey, and spectroscopic observations of the $\mathrm{H} \alpha$ line performed with the $3.9 \mathrm{~m}$ Anglo-Australian Telescope. These observations revealed AX J0049.4-7323 during an anomalous outburst having remarkably different properties compared to the previous ones. In the $I$ band, it showed a longer rise timescale ( $\sim 60$ days instead of $1-5$ days) and a longer decay timescale. At the peak of the outburst, it showed a sudden increase in luminosity in the $I$ band, corresponding to the onset of the X-ray outburst. The monitoring of the $\mathrm{H} \alpha$ emission line showed a fast and highly variable profile composed of three peaks with variable reciprocal brightness. To our knowledge, this is the second observation of a variable three-peak $\mathrm{H} \alpha$ profile of a Be/X-ray binary, after $\mathrm{A} 0535+26$. We interpreted these results as a circumstellar disc warped by tidal interactions with the neutron star in a high eccentricity orbit during its periastron passage. The fast jump in optical luminosity at the peak of the outburst and the previous asymmetric outbursts might be caused by the reprocessing of the X-ray photons in the circumstellar disc or the tidal displacement of a large amount of material from the circumstellar disc or the outer layers of the donor star during the periastron passage of the neutron star, which led to an increase in size of the region emitting in the $I$ band. Further multi-wavelength observations are necessary to discriminate among the different scenarios proposed to explain the puzzling optical and X-ray properties of AX J0049.4-7323.
\end{abstract}

Key words. stars: neutron - stars: emission-line, Be - X-rays: binaries - X-rays: individuals: AX J0049.4-7323

\section{Introduction}

$\mathrm{Be} / \mathrm{X}$-ray binaries (Be/XRBs, see Reig 2011 for a review) usually contain a neutron star (NS) or, rarely, a black hole (Casares et al. 2014 and e.g. Brown et al. 2018) accreting material from an early-type Oe-Be star. Oe-Be stars are O-B type stars of luminosity class III-V which, at some stages in their lives, show emission lines in their spectra (mostly in the Balmer and Paschen series) and an enhanced infrared (IR) radiation called IR excess (Waters et al. 1989). These features in their spectra are produced in a disc of dense gas lying on the equatorial plane of the donor star, called the circumstellar disc. The best-studied emission line of $\mathrm{Be}$ stars is the $\mathrm{H} \alpha$ line. Its morphology and variability give fundamental information about the structure, orientation, and evolution of the circumstellar disc (see e.g. Hanuschik et al. 1995 and Rivinius et al. 2013 for a recent review).

The optical luminosity of $\mathrm{Be} / \mathrm{XRBs}$ is dominated by the radiation coming from the surface of the Be star and the circumstellar disc (e.g. van Paradijs et al. 1998). Usually, their optical light curves show super-orbital $(\gtrsim 100 \mathrm{~d})$ irregular or quasi-periodic variability, with typical amplitudes in the $I$ band of $\Delta m_{I} \lesssim 0.6$, which are believed to be related to the formation and depletion of the circumstellar disc (Rajoelimanana et al. 2011, Reig \& Fabregat 2015 and references therein). The variability characteristics differ from source to source. They depend not only on the intrinsic properties of each source, but also on the orientation of the circumstellar disc with respect to the line of sight (e.g. Haubois et al. 2012). A small fraction of Be/XRBs shows orbital periodicities with amplitudes $\Delta m_{I} \approx 0.01-0.2$. This variability is usually ascribed to perturbations of the circumstellar disc produced by the orbiting NS. Some Be/XRBs show a fast rise-exponential decay (FRED) profile, which in some cases is associated with the orbital period. Bird et al. (2012) proposed using the "FRED-iness" of these profiles as an indicator to distinguish between orbital periods and other mechanisms like aliased radial and non-radial pulsations and long-term variability of the circumstellar disc. Despite the efforts and the valuable results obtained so far, a detailed model to explain the mechanism producing the FRED orbital modulation observed in some $\mathrm{Be} / \mathrm{XRB}$ is still missing.

As the name suggests, the main observational property that characterises the class of $\mathrm{Be} / \mathrm{XRBs}$ is their bright $\mathrm{X}$-ray 


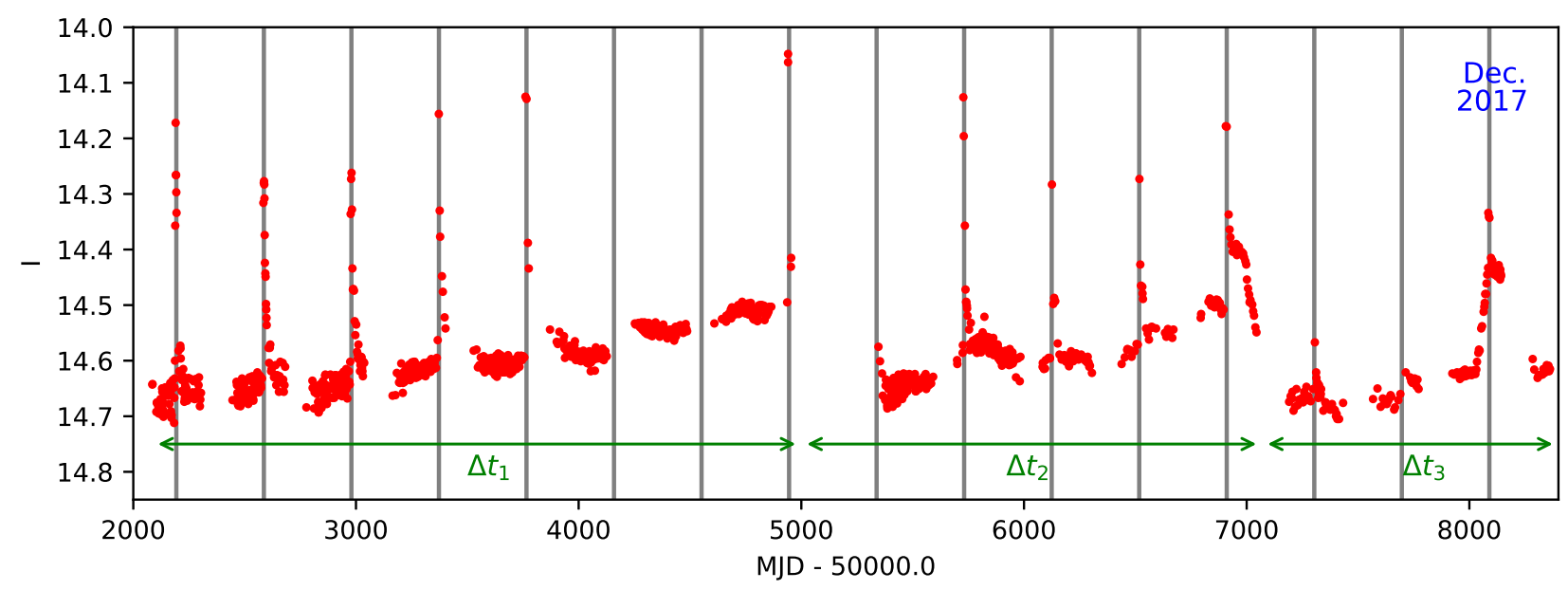

Fig. 1. OGLE light curve of AX J0049.4-7323 from June 2001 to September 2018. The grey vertical lines show the times of the peak of the optical outbursts according to the ephemeris calculated by Schmidtke et al. (2013). The green arrows at the bottom of the figure show the three epochs of gradual brightening of the basal emission (see Sect. 3.1).

emission. It is caused by the accretion of the dense material of the circumstellar disc on the compact object (Maraschi et al. 1976). These episodes of high accretion are sporadic, can last several weeks, and can reach X-ray luminosities of $\approx 10^{39} \mathrm{erg} \mathrm{s}^{-1}$ (e.g. Reig 2011; Townsend et al. 2017). Based on observations and theoretical models, it has recently been argued that the primary properties of the X-ray outbursts observed in most of Be/XRBs (e.g. the point of their onset along the orbit, their duration, the outburst rates, the X-ray luminosity and its variability) depend on the complicated interaction between the compact object and the circumstellar disc, whose structure can on some occasions deviate significantly from a symmetric geometry (Laplace et al. 2017; Negueruela et al. 2001; Reig et al. 2007; Moritani et al. 2013, Martin et al. 2011, 2014a,b; Okazaki et al. 2002). To understand the properties of the optical and X-ray outbursts and the mechanisms which trigger them, it is of great importance to collect information about the gas outflow from the Be star of these binary systems through multi-wavelength observations in X-ray and optical bands.

One of the few remarkable exceptions to the typical lowamplitude variability observed in Be/XRBs is AX J0049.4-7323 (SXP 756), which shows relatively short ( 30 days), bright $\left(\Delta m_{I} \approx 0.5\right)$, and periodic outbursts $\left(P_{\text {orb }}=393.1 \pm\right.$ $0.4 \mathrm{~d}$ Schmidtke et al. 2013) that are simultaneous with X-ray outbursts, with a periodicity that is thought to correspond to the orbital period of the system (Cowley \& Schmidtke 2003; Coe \& Edge 2004; Laycock et al. 2005; Galache et al. 2008). AX J0049.4-7323 is located in the Small Magellanic Cloud (SMC) and is composed of a $\sim 750 \mathrm{~s}$ pulsar (Yokogawa et al. 2000) orbiting around a O9.5-B0.5 III-V star (Edge \& Coe 2003; McBride et al. 2008). The photometric light curves based on the data from the MAssive Compact Halo Object Project (MACHO; Alcock et al. 1997) and the Optical Gravitational Lensing Experiment (OGLE; Sect. 2.3) of AX J0049.4-7323 presented by Rajoelimanana et al. (2011) showed that the amplitude of the outbursts vary and their peak luminosities increase with the optical emission between outbursts (hereafter basal emission). MACHO light curves in the $V$ and $R$ bands show that AX J0049.4-7323 becomes redder during most outbursts, suggesting that the emitting region gets cooler (Cowley \& Schmidtke 2003). The optical light curve folded at the orbital period shows a sharp and asymmetric peak ( $\Delta t \approx 30-40 \mathrm{~d}$ ), making it one of the best examples of a FRED profile (Rajoelimanana et al. 2011; Bird et al. 2012; Coe \& Edge 2004). Cowley \& Schmidtke (2003) found a quasiperiodic variability in the optical band of AX J0049.4-7323, on a timescale of $\sim 11 \mathrm{~d}$. They suggested that the observed modulation might be associated with the rotation of the disc of the $\mathrm{Be}$ star. Then Coe \& Edge (2004) proposed that the $\sim 11 \mathrm{~d}$ periodicity might be induced by the periastron passage of the NS which disturbs the geometry of the circumstellar disc. Schmidtke et al. (2013) proposed that the $\sim 11 \mathrm{~d}$ signal might actually be an alias produced by a signal at $\sim 0.917 \mathrm{~d}$. The light curve folded at this latter period shows a sinusoidal modulation, which is consistent with the expected optical variability due to non-radial pulsation of a Be star.

A spectroscopic study of the donor star of AX J0049.4-7323 carried out during an optical outburst (on 2001 November 6-12), showed a double-peaked $\mathrm{H} \alpha$ emission profile, indicating the presence of a circumstellar disc not observed face-on (Edge \& Coe 2003). The optical outburst was accompanied by an X-ray outburst detected by RXTE (Coe \& Edge 2004; Ducci et al. 2018).

Since its discovery, AX J0049.4-7323 has been observed in $\mathrm{X}$-rays with several satellites. As mentioned above, it shows periodic X-ray outbursts with peak luminosities of $\approx 10^{37} \mathrm{erg} \mathrm{s}^{-1}$ which are in phase with the optical outbursts, thought to occur near periastron (Coe \& Edge 2004; Laycock et al. 2005; Galache et al. 2008). AX J0049.4-7323 shows high X-ray variability along the orbit, spanning more than three orders of magnitude, with high luminosity states $\left(L_{\mathrm{x}} \geq 5 \times 10^{35} \mathrm{erg} \mathrm{s}^{-1}\right)$ far from periastron, and possibly two cases of anomalous fast variability (Ducci et al. 2018). The enhanced X-ray luminosity observed far from periastron during some orbital cycles might be due to the NS experiencing long periods of high accretion rate all along its orbit, likely caused by an extended and warped circumstellar disc or some other unknown mechanism (Coe \& Edge 2004; Ducci et al. 2018).

The optical and X-ray variability of AX J0049.4-7323 is unusual and still poorly understood. Therefore, we planned a monitoring campaign at different wavelengths, performed during the last outburst of December 2017, to investigate it more thoroughly. We made use of X-ray data from the Neil Gehrels Swift Observatory (Swift), simultaneously with spectroscopic and photometric observations in optical/UV bands with the 


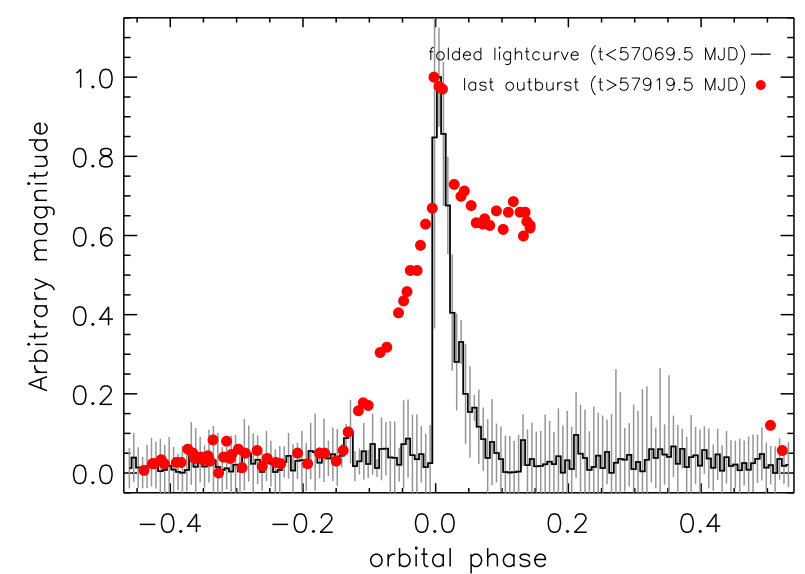

Fig. 2. Black line: folded OGLE light curve of AX J0049.4-7323 obtained using data before $t \leq 57069.5 \mathrm{MJD}$. Red points: OGLE outburst observed in December 2017. Both light curves are folded on the 393.1 d period (Schmidtke et al. 2013).

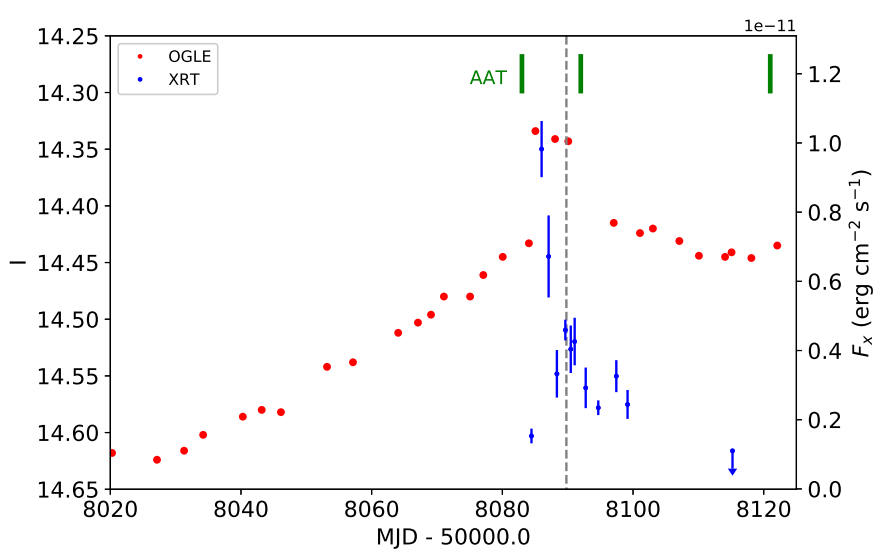

Fig. 3. Magnified view of the December 2017 outburst seen by OGLE (red points). Superimposed are the $0.3-8 \mathrm{keV}$ fluxes from the Swift/XRT monitoring of AX J0049.4-7323 (blue points), and the times of the AAT observations (green bars). The grey vertical dashed line shows the expected time of the peak of the optical outburst calculated with the ephemeris of Schmidtke et al. (2013).

Anglo-Australian Telescope (AAT) and Swift/UVOT, especially focused on the study of the variability of the $\mathrm{H} \alpha$ line, which provided with us important information about the properties of the circumstellar disc surrounding the system. In addition, we used the data from the OGLE survey collected over the last 17 years (Fig. 2). The results of these observations are presented in Sect. 3 and discussed in Sect. 4.

\section{Data analysis}

\subsection{Swift}

Table 1 shows the log of the Swift (Gehrels et al. 2004) observations analysed in this paper. These data were obtained as a monitoring program of 12 observations carried out near the expected outburst, based on the ephemeris calculated by Schmidtke et al. (2013). We processed and analysed X-ray Telescope (XRT; Burrows et al. 2005) data using the standard software $\left(\right.$ FTOOLS $^{1}$ v6.24) and calibration $\left(\mathrm{CALDB}^{2}\right.$

\footnotetext{
1 https://heasarc.gsfc.nasa.gov/docs/software/ lheasoft/ftools/

2 https://heasarc.gsfc.nasa.gov/docs/heasarc/caldb/ caldb_intro.html
}

20180411). XRT data were processed and filtered using XRTPIPELINE (v0.13.4). Source events were extracted from a circular region with a radius of 10 pixels $\left(1\right.$ pixel $\left.=2.36^{\prime \prime}\right)$, while the background was extracted from an annular region centred at the source position, with an inner radius of 30 pixels and an external radius of 70 pixels. The data were not affected by pile-up. The XRT light curve was corrected for point spread function losses, vignetting, and then background subctracted. Ancillary response files were created with the task XRTMKARF. The spectrum was extracted from all the datasets (except for the last observation, where the source was not detected) of Table 1 combined and analysed using XSPEC (v12.10.0c, Arnaud et al. 1996).

The UV/Optical Telescope (UVOT, Roming et al. 2005) observed AX J0049.4-7323 simultaneously with XRT, using six filters: $(V \approx 5468 \AA, B \approx 4392 \AA, U \approx 3465 \AA, w 1 \approx 2600 \AA$, $m 2 \approx 2246 \AA, w 2 \approx 1928 \AA$ ). The data analysis was performed using the tasks UVOTIMSUM and UvOTSOURCE. To calculate the magnitude, we adopted a circular region with radius of $5^{\prime \prime}$ centred on the source position and a circular region with a radius of $10^{\prime \prime}$ for the background.

\subsection{Anglo-Australian Telescope}

Three optical spectroscopic observations of AX J0049.4-7323 were performed with the $3.9 \mathrm{~m}$ Anglo-Australian Telescope (AAT) during the nights of 2017 November 26, December 5, and 2018 January 3. Observations were carried out with the High Efficiency and Resolution Multi-Element Spectrograph (HERMES; Sheinis 2016). HERMES is a four-channel fibre-fed spectrograph with high resolution $(R=\lambda / \Delta \lambda \sim 28000)$ and multi-object capability, sensitive in the range $471.5-788.7 \mathrm{~nm}$. The source was observed with a $3 \times 2400 \mathrm{~s}$ of good exposure time and a 2 arcsec slit width. All runs were performed with the 2785 line/mm cross-dispersing grating. Each channel has individual CCD cameras, each of $4 k \times 4 k$, of the E2V family with $15 \mu \mathrm{m}$ pixels, operating at about $170 \mathrm{~K}$. We reduced all data using a standard $2 \mathrm{drfr}$ software package ${ }^{3}$ dedicated to reducing HERMES data, and standard Image Reduction and Analysis Facility (IRAF) ${ }^{4}$ packages (version 2.16). Images were bias- and flatfield-corrected, and wavelength calibration using arc lamp spectra (thorium-argon) was applied.

\subsection{OGLE}

OGLE is a photometric sky survey that began in April 1992 (Udalski et al. 2015). It monitors regularly the Galactic Bulge, part of the Galactic disc, and the Magellanic Clouds, and it provides photometry in the $I$ band with uncertainties of about $5 \times 10^{-3}$ mag for the SMC fields (an analysis of the OGLE errors can be found in Skowron et al. 2016). During the first phase (1992-1995), the main goal of the project was the detection of gravitational microlensing events to constrain the nature of dark matter (e.g. Paczynski 1986). However, thanks to the constant monitoring of about a billion stars, OGLE proved to be a useful project for the study of numerous other physical phenomena. These include the study of the optical variability of Be/XRBs (Rajoelimanana et al. 2011; Bird et al. 2012; Schmidtke et al. 2013). The photometric data used in this paper have been retrieved from the OGLEIII and IV X-ray variables OGLE

\footnotetext{
3 https://www.aao.gov.au/science/software/2dfdr 4 iraf.noao.edu
} 
monitoring (XROM, Udalski 2008) web $\operatorname{sites}^{5,6}$ and have been corrected to the standard $I$-band system.

\section{Results}

\subsection{Optical photometry}

Figure 1 shows that the optical outbursts observed in the OGLE survey are superimposed on three epochs of gradual brightening of the basal emission $\left(\Delta t_{1}=52085.4-55000 \mathrm{MJD} ; \Delta t_{2}=\right.$ $\left.55000-57069 \mathrm{MJD} ; \Delta t_{3}>57069 \mathrm{MJD}\right)$. From the outburst at $\sim 56800 \mathrm{MJD}$, the main features characterising the optical variability of AX J0049.4-7323 changed significantly. The most important new pieces of information after this event emerging from Fig. 1 are the following:

- the decay tail of the outburst observed at $\sim 56800$ MJD is much longer ( $\sim 140$ days) than those previously observed and its shape is also peculiar as it shows a hump during the decay phase;

- after this anomalous outburst, the basal luminosity decreased to $I \approx 14.6$, i.e. about $0.15 \mathrm{mag}$ fainter than the magnitude prior to the outburst. Since then, and until the last outburst, AX J0049.4-7323 showed a rebrightening of the basal emission similar to that previously observed;

- in the two successive orbital cycles, the amplitude of the optical outbursts were much lower than in the past. The shape of the alleged outburst during the second cycle was peculiar; it was similar to a step. However, it is important to note that there is a gap of about 24 days in the data at the expected peak position;

- The last outburst of Fig. 1, which peaks at 58 090 MJD, shows a longer rise timescale (about 60 days) compared to the previous ones (the outbursts with more frequent sampling show a rise time of about 2-5 days) and also a longer decay timescale. The lack of data between $~ 58142$ MJD and $~ 58284$ MJD does not allow us to estimate its duration. Despite this gap of data, we note that the decay phase of this outburst shows a peculiar hump, already observed in the bright outburst observed at 56 800 MJD.

The anomalous shape of the December 2017 ( $\approx 58090$ MJD) outburst is highlighted in Fig. 2, where it is compared to the FRED profile of the OGLE light curve folded on the $393.1 \mathrm{~d}$ period. We created the folded light curve from the detrended OGLE light curve obtained from two polynomial functions (three orders each, in the ranges $\Delta t_{1}$ and $\Delta t_{2}$ ) fitted to the OGLE data where the periods of the outbursts were removed, and then subtracted from the complete light curve, i.e. the one which includes the outbursts. Figure 3 shows that during the December 2017 outburst, the I-band luminosity of AX J0049.4-7323 increased almost constantly up to $t \approx 58085 \mathrm{MJD}$, where the source showed a sudden increase in the luminosity that was simultaneous (within $\sim 1$ day) with the onset of the X-ray outburst observed by Swift/XRT.

Figure 4 shows the colour-magnitude diagram $U-I$ versus $I$ obtained from the UVOT and OGLE data of the last outburst (blue, see Fig. 5) and of two previous observations carried out far from periastron (yellow: $\mathrm{MJD}_{1} \approx 55797.77 ; \mathrm{MJD}_{2}=57289.16$, corresponding to the orbital phases $\phi_{1}=0.17$ and $\phi_{2}=0.96$; these two measurements were reported in Ducci et al. 2018). The $I$ magnitudes of Fig. 4 corresponding to the $U$ points 4 , 5 , and 7 , were obtained from interpolation. The plot shows that

\footnotetext{
5 http://ogle.astrouw.edu.pl/ogle3/xrom/xrom.html

6 http://ogle.astrouw.edu.pl/ogle4/xrom/xrom.html
}

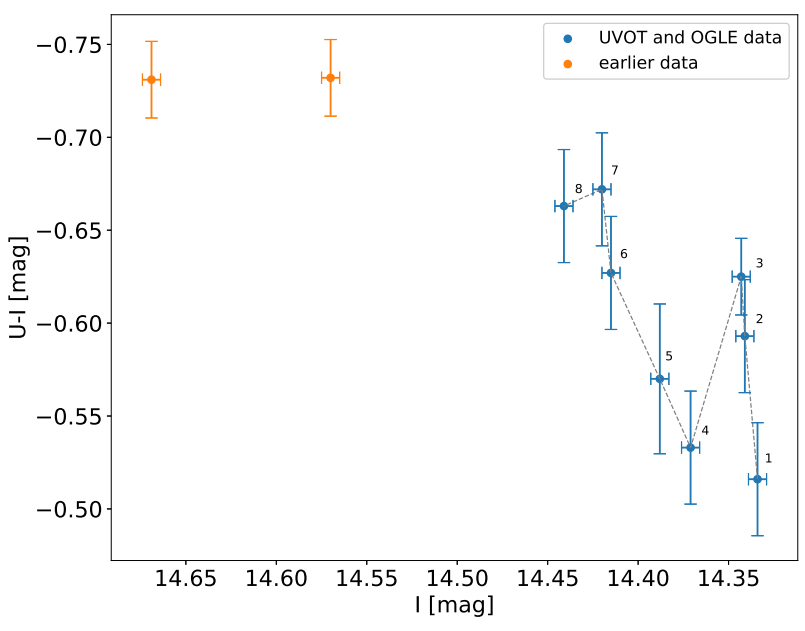

Fig. 4. Colour magnitude diagram of AX J0049.4-7323 based on UVOT and OGLE data of the December 2017 outburst (blue points) and in two previous observations carried out far from periastron (yellow points). Labels from 1 to 8 show the timeline of the observations during the December 2017 outburst (see also Fig. 5).

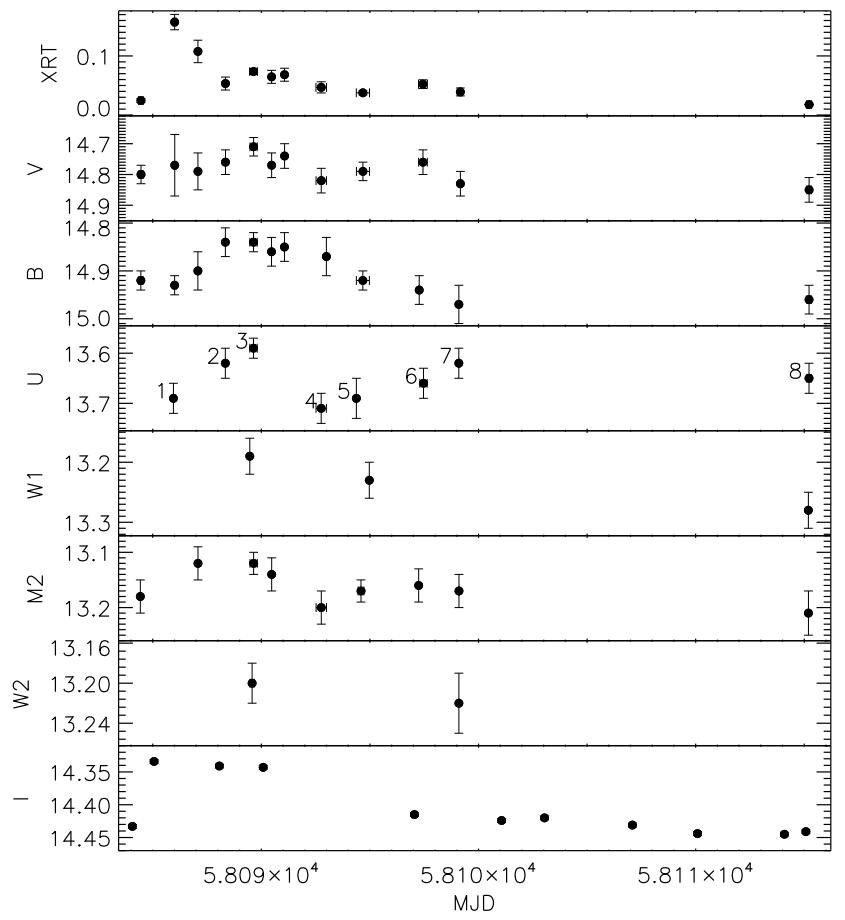

Fig. 5. Swift/XRT (0.3-10 keV), UVOT ( $V, B, U, W 1, M 2, W 2$ bands), and OGLE ( $I$ band) light curves of AX J0049.4-7323 obtained during the December 2017 monitoring campaign. Error bars indicate the $1 \sigma$ statistical uncertainties. OGLE error bars and symbols are similar in size. UVOT systematic errors are comparable to the statistical ones.

the source reddens when it goes in outburst and it cools during the outburst. This behaviour was previously observed by Cowley \& Schmidtke (2003) in AX J0049.4-7323 using $V, R$, and $I$ magnitudes provided by the MACHO and OGLE-II survey, and it was explained with a brightening of the circumstellar disc during the outburst.

\subsection{Swift/XRT}

The average XRT spectrum during the outburst can be well fitted $\left(\chi_{v}^{2}=1.160,41\right.$ d.o.f. $)$ with an absorbed $\left(N_{\mathrm{H}}=4.8_{-0.9}^{+1.1} \times\right.$ 
Table 1. Summary of the Swift/XRT observations.

\begin{tabular}{llll}
\hline \hline ObsID & Start time (UT) & End time (UT) & $\begin{array}{l}\text { Exp. } \\
\text { (s) }\end{array}$ \\
\hline 00010380001 & $2017-11-27$ 08:23:08 & $2017-11-27$ 13:29:52 & 4947 \\
00010380002 & $2017-11-28$ 22:29:48 & $2017-11-2901: 54: 21$ & 1978 \\
00010380004 & $2017-11-30$ 01:51:00 & $2017-11-30$ 01:59:54 & 534 \\
00010380005 & $2017-12-0108: 09: 58$ & $2017-12-0108: 27: 53$ & 1076 \\
00010380006 & $2017-12-02 ~ 11: 12: 03$ & $2017-12-0219: 30: 52$ & 7023 \\
00010380007 & $2017-12-03$ 11:09:51 & $2017-12-03 ~ 11: 26: 54$ & 1023 \\
00010380008 & $2017-12-04$ 01:27:31 & $2017-12-0401: 45: 52$ & 1101 \\
00010380009 & $2017-12-0512: 23: 38$ & $2017-12-05 ~ 23: 59: 54$ & 1086 \\
00010380010 & $2017-12-0708: 58: 56$ & $2017-12-0723: 33: 53$ & 6609 \\
00010380012 & $2017-12-1005: 44: 59$ & $2017-12-1015: 26: 53$ & 1667 \\
00010380013 & $2017-12-1202: 12: 12$ & $2017-12-1205: 37: 54$ & 1617 \\
00010380014 & $2017-12-2802: 49: 50$ & $2017-12-2807: 51: 54$ & 1259 \\
\hline
\end{tabular}

$10^{21} \mathrm{~cm}^{-2}$; calculated using tbabs model in xspec, Wilms et al. 2000) power law $\left(\Gamma=0.66_{-0.09}^{+0.11}\right)$. The parameters of the fit are listed in Table 2, and the average spectrum is shown in Fig. 6 (all errors quoted at the $1 \sigma$ confidence level). Ducci et al. (2018) showed an anti-correlation between $\Gamma$ and the flux using previous X-ray observations of AX J0049.4-7323. Considering the new measure shown in Table 2, we increased the significance of this anti-correlation: the Pearson's linear coefficient becomes $r=-0.85$ and the null hypothesis probability in the $\log _{10} x-y$ space is $p=1.8 \%$. The spectral slope softens at lower fluxes following the relation $\Gamma=(-3.72 \pm$ $1.3) \times \log _{10} F_{\mathrm{x}}-(0.38 \pm 0.11)$, where the errors are quoted at the $1 \sigma$ confidence level. The anti-correlation between $\Gamma$ and the flux including the new Swift/XRT observation is shown in Fig. 7.

\section{3. $\mathrm{H} \alpha$ emission line}

The $\mathrm{H} \alpha$ line was observed three times, at the peak of the December 2017 outburst and during the fading phase, for about 40 days. The line profiles are shown in Fig. 8. Dealing with doublepeaked profiles, the equivalent width (EW) was calculated using the splot task of IRAF, which employs the method of direct integration of the flux across the feature. Moreover, double-peaked profiles have also been fitted using the deblending routine available in IRAF. The three peaks have been modelled with three Voigt functions, which fitted the line wings better than Gaussian functions, returning line centres and intensities of the three peaks above the continuum. By these, we calculated the separation between the red and blue peaks $\Delta V$. Following the methodology of Reig et al. (2010) and Malacaria et al. (2017), to derive the $\mathrm{H} \alpha$ line spectral parameters we iterated the fitting procedure twelve times for each double-peaked feature and sampled, at each iteration, a slightly different point of the continuum (whose definition is the main source of uncertainty during this process). Final values and errors of line parameters were calculated as the average and standard deviation over those iterated measures. The equivalent width $E W$ and the separation between red and blue peaks $\Delta V$ of each observation are given in Table 3 . The $\mathrm{H} \alpha$ line showed a high variability and a peculiar shape in all three observations. In particular, during observation A, carried out slightly before the peak of the outburst (see Fig. 1), the $\mathrm{H} \alpha$ profile showed a three-peak profile with a narrow central peak and two side peaks of about the same height $(V=R)$. Nine days later (observation B), when the optical emission from the source was fading, the $\mathrm{H} \alpha$ profile changed significantly: its intensity increased, and the height of the red peak became higher than the
Table 2. Best fit parameters of the Swift/XRT average spectrum.

\begin{tabular}{lc}
\hline \hline Parameter & Value \\
\hline$N_{\mathrm{H}}\left(10^{21}\right) \mathrm{cm}^{-2}$ & $4.8_{-0.9}^{+1.1}$ \\
$\Gamma$ & $0.66_{-0.09}^{+0.11}$ \\
$\chi_{v}^{2}$ (d.o.f.) & $1.160(41)$ \\
Absorbed flux & $3.55 \pm 0.14 \times 10^{-12}$ \\
Unabsorbed flux & $3.97_{-0.13}^{+0.15} \times 10^{-12}$ \\
\hline
\end{tabular}

Notes. Fluxes are in units of erg $\mathrm{cm}^{-2} \mathrm{~s}^{-1}$ in the $0.3-8 \mathrm{keV}$ energy range. Errors are quoted at the $1 \sigma$ confidence level.

blue peak $(V<R)$. The observed $V / R$ variability could be due to density perturbations in the circumstellar disc caused by the NS or to one-armed disc oscillations of the circumstellar disc similar to those observed in other Be stars (see e.g. Rivinius et al. 2013 and references therein). In observation $\mathrm{C}$, carried out about one month later, when AX J0049.4-7323 was much fainter in the $I$ band and not detected in X-ray, the $\mathrm{H} \alpha$ profile still shows a relatively high intensity compared to observation B and especially observation $\mathrm{A}$. The height of the blue and red peaks was much lower compared to the central peak (they almost disappeared).

\section{Discussion}

In Sect. 3.1 we showed that AX J0049.4-7323 experienced a change in its optical properties after the outburst observed at $\sim 56800$ MJD. The decrease in the $I$ magnitude after this outburst and the low amplitude of the two subsequent outbursts compared to the previous ones displayed by the system, might be due to a rapid depletion of the circumstellar disc (likely triggered by the tidal interaction with the NS) which then slowly formed again. Unfortunately, the binary system was not observed at other wavelengths during the anomalous outburst of $~ 56800 \mathrm{MJD}$ and the lack of $\mathrm{H} \alpha$ observations during and immediately after that event prevents us from testing our hypothesis.

The multi-wavelength monitoring campaign of AX J0049.4-7323 carried out in December 2017 revealed an anomalous outburst whose optical emission was characterised by rise and decay timescales longer than in the previous outbursts. As shown in Fig. 2, the outburst did not show the distinctive FRED profile observed in the past. The rise of the outburst can be divided in two parts. In the first part the flux increases slowly, starting from $t_{0} \approx 58020$ MJD (corresponding to the phase $\phi \approx-0.15$ in Fig. 2 ) and rising to $t_{1}=58085 \mathrm{MJD}$. The rise timescale of this part of the outburst is relatively fast compared to the typical timescales of the formation of circumstellar discs in Be/XRBs (see e.g. Haubois et al. 2012), but it is roughly consistent with those seen in some of them (e.g. Kizıloğlu et al. 2009; Rajoelimanana et al. 2011). We suggest that it might be due to an increment in the size of the circumstellar disc triggered by the tidal interactions of a NS in an eccentric orbit that approaches periastron.

In the second part of the light curve, from $t_{1}$ to the peak, AX J0049.4-7323 displays a steep jump in the optical brightness synchronised with the onset of the X-ray outburst. The fast increase in luminosity at $t_{1}$ is similar to that of the FRED outbursts observed prior to $\approx 56800 \mathrm{MJD}$, though with much lower amplitude, and might suggest that another mechanism (likely triggered by the X-ray outburst) could be the cause of the bright peak of this outburst and also the previous asymmetric outbursts. 


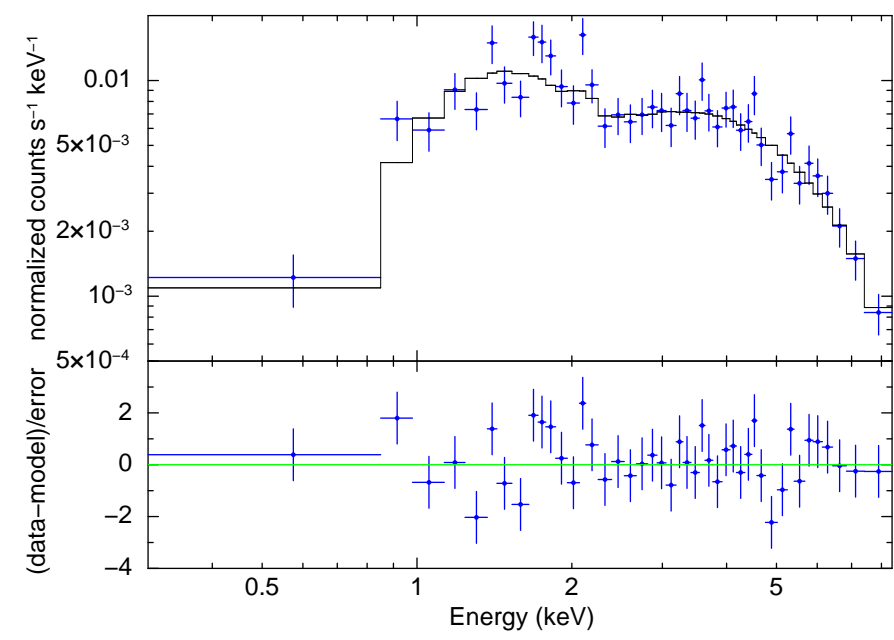

Fig. 6. Average Swift/XRT spectrum of the December-January 2018 campaign fitted with an absorbed power law (see Table 2). The residuals of the fit are shown in the bottom panel.

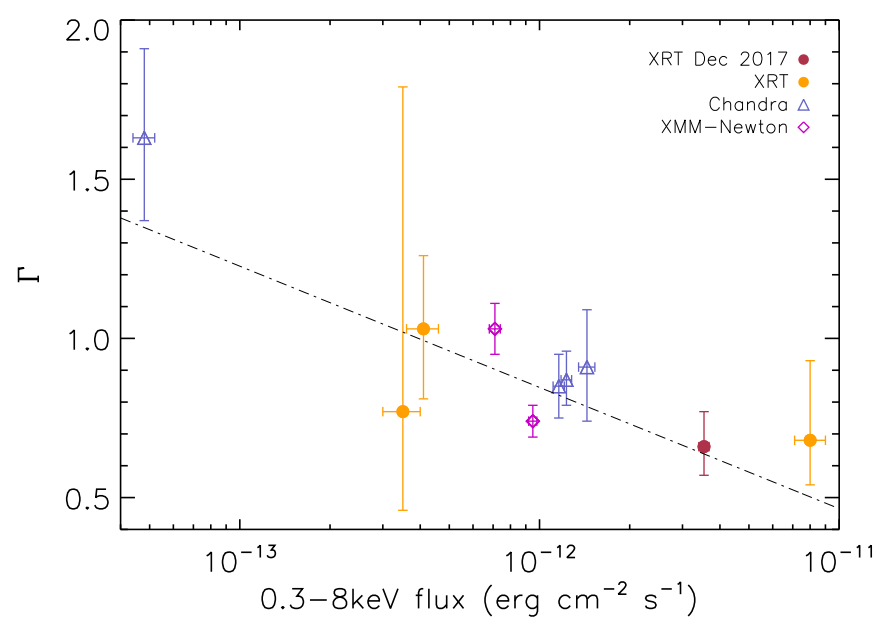

Fig. 7. Photon index $(\Gamma)$ as a function of the absorbed flux. Errors are quoted at the $1 \sigma$ confidence level. The plotted values correspond to those listed in Table 3 of Ducci et al. (2018; yellow circles, blue triangles, magenta diamonds) and Table 2 of this paper (red circle).

The current explanation for the FRED-shaped optical outbursts simultaneous with the X-ray outbursts was proposed by Cowley \& Schmidtke (2003) and Coe \& Edge (2004). They suggested that the optical outbursts were caused by the disruption of the circumstellar disc caused by its interaction with the NS. They also pointed out that a similar outburst behaviour was observed in another Be/XRB, A0538-66. In this system, an optical variability spanning more than 2 magnitudes was observed simultaneously with bright X-ray outbursts $\left(L_{\mathrm{x}} \approx 10^{39} \mathrm{erg} \mathrm{s}^{-1}\right.$, Charles et al. 1983 and references therein). Charles et al. (1983), Maraschi et al. (1983), and Apparao \& Tarafdar (1988) suggested that the bright optical outbursts of A0538-66 were produced by the reprocessing of the X-ray photons emitted by the NS in the circumstellar envelope around the binary system. A similar mechanism could explain the asymmetric outbursts observed in AXJ0049.4-7323 prior to $\approx 56800 \mathrm{MJD}$ and the sudden increase in luminosity at $t_{1}$ observed in the December 2017 outburst. A similar possibility was also considered by McGowan et al. (2008) for the optical variability shown by SXP 46.6, an accreting pulsar in the SMC. Alternatively, these fast outbursts might be caused by the tidal displacement
Table 3. Spectral parameters of the $\mathrm{H} \alpha$ emission line.

\begin{tabular}{llcc}
\hline \hline Obs. & Date & $\begin{array}{c}E W \\
(\AA)\end{array}$ & $\begin{array}{c}\Delta V \\
\left(\mathrm{~km} \mathrm{~s}^{-1}\right)\end{array}$ \\
\hline A & 2017-Nov.-26 & $-17.8 \pm 3.0$ & $280.3 \pm 20.9$ \\
$\mathrm{~B}$ & 2017-Dec.-05 & $-24.3 \pm 4.1$ & $262.1 \pm 4.5$ \\
$\mathrm{C}$ & 2018-Jan.-03 & $-20.8 \pm 3.9$ & $220.3 \pm 3.3$ \\
\hline
\end{tabular}

Notes. Errors quoted at the $1 \sigma$ confidence level.

of a large amount of material from the circumstellar disc or the outer layers of the donor star during the periastron passage of the NS, which then translates to a significant increase in the size of the emitting region and hence in the optical luminosity.

In Sect. 3.3 we presented the results of three observations of the $\mathrm{H} \alpha$ line carried out during the December 2017 outburst. Previously, the $\mathrm{H} \alpha$ profile of AX J0049.4-7323 was observed only once by Edge \& Coe (2003), during another optical outburst (at the orbital phase $\phi \approx 0.07$, according to the ephemeris of Schmidtke et al. 2013). In that observation it showed the typical double peak seen in many other Be stars with a circumstellar disc observed at a high inclination angle. In our observations, the $\mathrm{H} \alpha$ profile showed three peaks with variable reciprocal intensity. Although with remarkably different properties from those of AX J0049.4-7323, only a few other Be/XRBs showed anomalous $\mathrm{H} \alpha$ profiles. Among these, two interesting cases are 4U0115+63 and A0535+26. Negueruela et al. (2001) reported the observations of a succession of singlepeaked and shell profiles in the emission lines of $4 \mathrm{U} 0115+63$ on a timescale of several months. Shell profiles are usually associated with Be discs seen edge-on, while single-peaked profiles are observed in pole-on Be discs. They interpreted their observations with a highly tilted warped precessing circumstellar disc. The large variability of the $\mathrm{H} \alpha$ emission line of 4U $0115+63$ was associated with the onset of giant X-ray outbursts. Moritani et al. (2011, 2013), studied the $\mathrm{H} \alpha$ line profile variability of A0535+26 from 2009 to 2012. During this period, the source displayed several X-ray outbursts. In 2009, during a giant X-ray outburst, the line profile showed three peaks. They interpreted these features as the result of a precessing warped circumstellar disc. Each of the three peaks was produced in different regions of the warped disc observed from different angles. We suggest that both the peculiar shape and the high variability of the $\mathrm{H} \alpha$ profile of AX J0049.4-7323 presented in this work might be interpreted with a scenario similar to that proposed by Moritani et al. (2011, 2013) for A0535+26, namely with perturbations in the circumstellar disc driven by radiation or by tidal interactions with the NS in a high eccentricity orbit during its periastron passage. Similar to what was done by Moritani et al. (2011), we used the calculations presented by Porter (1998) to estimate the timescale associated with the precession of a radiatively induced warped disc around a Be star. We found that a radiatively driven warped disc around AX J0049.4-7323 would have a precessing timescale ranging from one to five months. The lower limit of about 30 days, obtained assuming the stellar properties of a $09.5 \mathrm{~V}$ star, is compatible with the observed timescale. Another way to warp the disc is through tidal interactions with the NS. Martin et al. (2011, 2014a,b) showed, with numerical simulations, that if the circumstellar disc of a Be star and the orbital plane of the NS are misaligned, the tidal torque exerted by the NS on the disc can warp it and make it more elongated. Interestingly, an extended and warped circumstellar disc, 


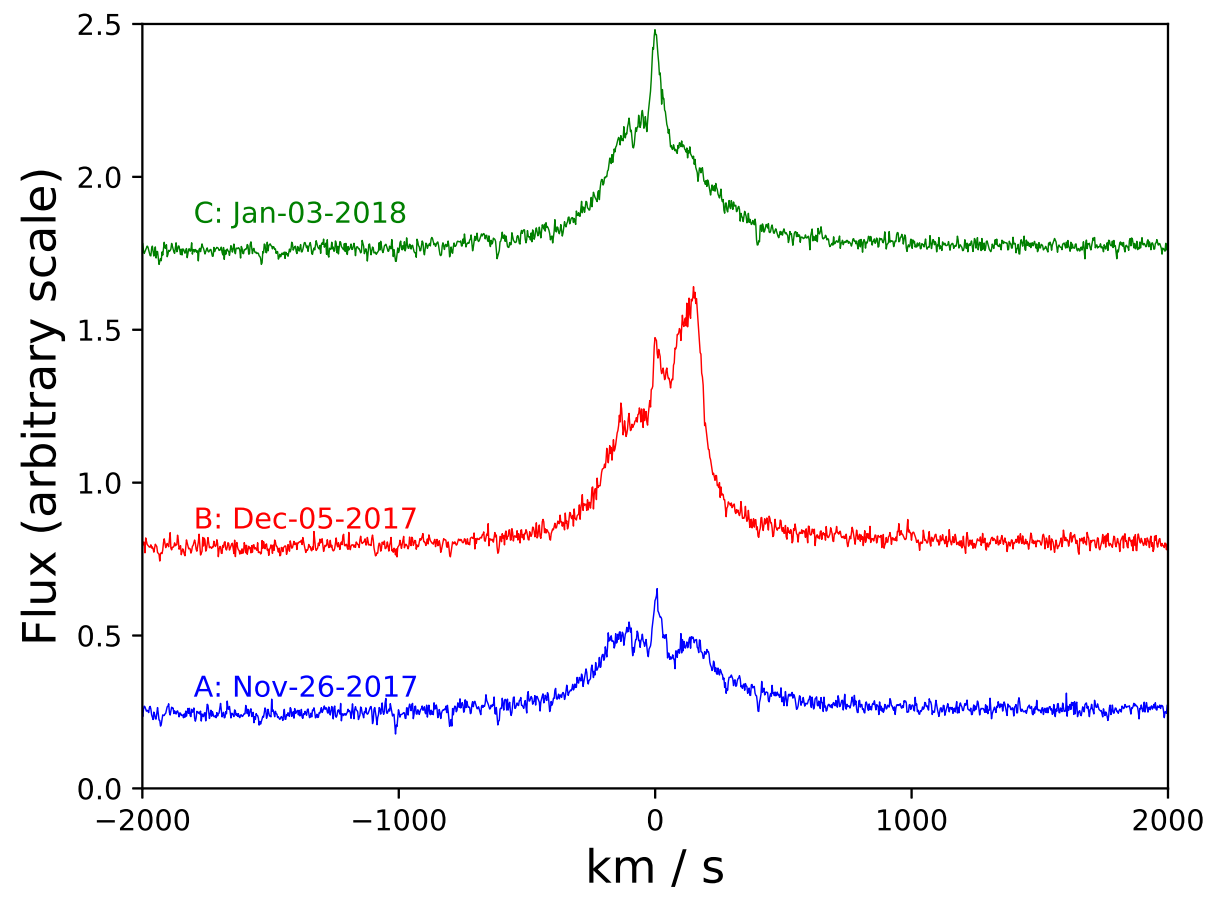

Fig. 8. H $\alpha$ emission lines of AX J0049.4-7323 during the December 2017 outburst. The velocity scale is normalised to the recession velocity of the SMC of $\approx 145.6 \mathrm{~km} \mathrm{~s}^{-1}$ (Harris \& Zaritsky 2006). slightly misaligned with respect to the orbital plane, would also explain the high X-ray variability of AX J0049.4-7323 observed far from periastron reported in Ducci et al. (2018).

An alternative explanation is that the complicated shape of the $\mathrm{H} \alpha$ line of AX J0049.4-7323 is caused by the superposition of two contributions from the circumstellar disc and from an accretion disc around the NS, with different reciprocal orientations. Kızıloğlu et al. (2009) suggested that accretion discs around a NS in a $\mathrm{Be} / \mathrm{XRB}$ might contribute to the $\mathrm{H} \alpha$ line. This line has been used to reveal the presence of an accretion disc in other X-ray binary systems such as SS 433 (Bowler 2010), IGR J17329-2731 (Bozzo et al. 2018), and IGR J00291+5934 (Lewis et al. 2010). It is important to note that in this framework some problems would emerge for the case of AX J0049.4-7323 that require further clarification. The central peak was not present during a previous bright X-ray and optical outburst (November 2001, see Sect. 1), when the $\mathrm{H} \alpha$ line was observed to have the typical double-peak profile produced by the circumstellar discs seen in the spectra of many Be stars. The absence of the central emission line in the previous outburst would suggest that this component is caused by a transient accretion disc. It is worth noting that the central peak of the $\mathrm{H} \alpha$ line observed during the December 2017 outburst has an EW of about $-0.9 \AA$, which is strikingly similar to that observed for the single-peaked $\mathrm{H} \alpha$ line from the face-on accretion disc of the Xray binary MAXI J1836-194 (Russell et al. 2014). Although it is an attractive scenario, due to the lack of more detailed observations of AX J0049.4-7323 it is not possible to go beyond these speculations.

\section{Conclusions}

We presented the results from a multi-wavelength monitoring campaign of the Be/XRB AX J0049.4-7323 during the outburst of December 2017 in X-ray and optical. We observed the binary system during an anomalous optical outburst characterised by longer rise and decay timescales than in the previous outbursts, and without the characteristic FRED profile observed previously. Near the peak of the outburst, the source showed a sudden increase in the flux in X-ray and $I$ bands. We proposed that the properties of the $\mathrm{H} \alpha$ line and the variability of AX J0049.4-7323 in the $I$ band indicate the presence of a highly perturbed circumstellar disc, likely warped and elongated by the tidal interaction with a NS in a high eccentricity orbit during the periastron passage. We also suggested that the X-ray reprocessing of X-ray photons in the circumstellar disc might be the cause of the asymmetric outbursts observed prior to $\approx 56800 \mathrm{MJD}$ and the jump in optical luminosity at the peak of the December 2017 outburst. We proposed an alternative scenario, where also an accretion disc around the NS contributes to the complicated shape of the $\mathrm{H} \alpha$ line. Although the monitoring campaign of the December 2017 outburst has revealed new interesting properties of AX J0049.4-7323, the information collected so far are insufficient to draw any firm conclusion about the peculiar optical and X-ray variability of AX J0049.4-7323. Therefore, new multiwavelength observations and the determination of the orbital parameters of the system, such as the eccentricity and the inclination angle between the orbital plane and the circumstellar disc, will be fundamental to answer some of the most important open questions about the puzzling properties of AX J0049.4-7323, with particular emphasis on the morphology of the gas envelope around AXJ0049.4-7323 and the mechanism responsible for the simultaneous "FRED" optical and X-ray outbursts.

Acknowledgements. We thank the anonymous referee for the constructive comments that helped to improve the paper. This paper is based in part on data from the Neil Gehrels Swift Observatory and on data acquired through the Australian Astronomical Observatory. We acknowledge the traditional owners of the land on which the AAT stands, the Gamilaraay people, and pay our respects to elders past and present. This work is supported by the Bundesministerium für Wirtschaft und Technologie through the Deutsches Zentrum für Luft und Raumfahrt (grant FKZ 50 OG 1602). P.R. acknowledges contract ASI-INAF I/004/11/0. C.M. is supported by an appointment to the NASA Postdoctoral Program at the Marshall Space Flight Center, administered by Universities Space Research Association under contract with NASA. The OGLE project has received funding from the National Science Centre, Poland, grant MAESTRO 2014/14/A/ST9/00121 to AU. J.L. is grateful for the support from the Chinese NSFC 11733009. 


\section{References}

Alcock, C., Allsman, R. A., Alves, D., et al. 1997, ApJ, 486, 697 Apparao, K. M. V., \& Tarafdar, S. P. 1988, ApJ, 335, 147

Arnaud, K. A. 1996, in Astronomical Data Analysis Software and Systems V, eds. G. H. Jacoby, \& J. Barnes, ASP Conf. Ser., 101, 17

Bird, A. J., Coe, M. J., McBride, V. A., \& Udalski, A. 2012, MNRAS, 423, 3663

Bowler, M. G. 2010, A\&A, 516, A24

Bozzo, E., Bahramian, A., Ferrigno, C., et al. 2018, A\&A, 613, A22

Brown, R. O., Ho, W. C. G., Coe, M. J., \& Okazaki, A. T. 2018, MNRAS, 477, 4810

Burrows, D. N., Hill, J. E., Nousek, J. A., et al. 2005, Space Sci. Rev., 120, 165

Casares, J., Negueruela, I., Ribó, M., et al. 2014, Nature, 505, 378

Charles, P. A., Booth, L., Densham, R. H., et al. 1983, MNRAS, 202, 657

Coe, M. J., \& Edge, W. R. T. 2004, MNRAS, 350, 756

Cowley, A. P., \& Schmidtke, P. C. 2003, AJ, 126, 2949

Ducci, L., Romano, P., Malacaria, C., et al. 2018, A\&A, 614, A34

Edge, W. R. T., \& Coe, M. J. 2003, MNRAS, 338, 428

Galache, J. L., Corbet, R. H. D., Coe, M. J., et al. 2008, ApJS, 177, 189

Gehrels, N., Chincarini, G., Giommi, P., et al. 2004, ApJ, 611, 1005

Hanuschik, R. W., Hummel, W., Dietle, O., \& Sutorius, E. 1995, A\&A, 300, 163

Harris, J., \& Zaritsky, D. 2006, AJ, 131, 2514

Haubois, X., Carciofi, A. C., Rivinius, T., Okazaki, A. T., \& Bjorkman, J. E. 2012, ApJ, 756, 156

Kızıloğlu, Ü., Özbilgen, S., Kızıloğlu, N., \& Baykal, A. 2009, A\&A, 508, 895

Laplace, E., Mihara, T., Moritani, Y., et al. 2017, A\&A, 597, A124

Laycock, S., Corbet, R. H. D., Coe, M. J., et al. 2005, ApJS, 161, 96

Lewis, F., Russell, D. M., Jonker, P. G., et al. 2010, A\&A, 517, A72

Malacaria, C., Kollatschny, W., Whelan, E., et al. 2017, A\&A, 603, A24

Maraschi, L., Treves, A., \& van den Heuvel, E. P. J. 1976, Nature, 259, 292

Maraschi, L., Traversini, R., \& Treves, A. 1983, MNRAS, 204, 1179

Martin, R. G., Pringle, J. E., Tout, C. A., \& Lubow, S. H. 2011, MNRAS, 416 2827

Martin, R. G., Nixon, C., Armitage, P. J., Lubow, S. H., \& Price, D. J. 2014a ApJ, 790, L34
Martin, R. G., Nixon, C., Lubow, S. H., et al. 2014b, ApJ, 792, L33

McBride, V. A., Coe, M. J., Negueruela, I., Schurch, M. P. E., \& McGowan, K. E. 2008, MNRAS, 388, 1198

McGowan, K. E., Coe, M. J., Schurch, M. P. E., et al. 2008, MNRAS, 384, 821

Moritani, Y., Nogami, D., Okazaki, A. T., et al. 2011, PASJ, 63, 25

Moritani, Y., Nogami, D., Okazaki, A. T., et al. 2013, PASJ, 65, 83

Negueruela, I., Okazaki, A. T., Fabregat, J., et al. 2001, A\&A, 369, 117

Okazaki, A. T., Bate, M. R., Ogilvie, G. I., \& Pringle, J. E. 2002, MNRAS, 337, 967

Paczynski, B. 1986, ApJ, 304, 1

Porter, J. M. 1998, A\&A, 336, 966

Rajoelimanana, A. F., Charles, P. A., \& Udalski, A. 2011, MNRAS, 413, 1600

Reig, P. 2011, APSS, 332, 1

Reig, P., \& Fabregat, J. 2015, A\&A, 574, A33

Reig, P., Larionov, V., Negueruela, I., Arkharov, A. A., \& Kudryavtseva, N. A. 2007, A\&A, 462, 1081

Reig, P., Zezas, A., \& Gkouvelis, L. 2010, A\&A, 522, A107

Rivinius, T., Carciofi, A. C., \& Martayan, C. 2013, A\&ARv, 21, 69

Roming, P. W. A., Kennedy, T. E., Mason, K. O., et al. 2005, Space Sci. Rev., 120,95

Russell, T. D., Soria, R., Motch, C., et al. 2014, MNRAS, 439, 1381

Schmidtke, P. C., Cowley, A. P., \& Udalski, A. 2013, MNRAS, 431, 252

Sheinis, A. I. 2016, in Ground-based and Airborne Instrumentation for Astronomy VI, Proc. SPIE, 9908, 99081C

Skowron, J., Udalski, A., Kozłowski, S., et al. 2016, Acta Astron., 66, 1 Townsend, L. J., Kennea, J. A., Coe, M. J., et al. 2017, MNRAS, 471, 3878 Udalski, A. 2008, Acta Astron., 58, 187

Udalski, A., Szymański, M. K., \& Szymański, G. 2015, Acta Astron., 65, 1 van Paradijs, J. 1998, NATO Advanced Science Institutes (ASI) Series C, eds. R. Buccheri, J. van Paradijs, \& A. Alpar, 515, 279

Waters, L. B. F. M. 1989, in Two Topics in X-Ray Astronomy, Volume 1: X Ray Binaries. Volume 2: AGN and the XRay Background eds. J. Hunt, \& B. Battrick, ESA SP, 296

Wilms, J., Allen, A., \& McCray, R. 2000, ApJ, 542, 914

Yokogawa, J., Imanishi, K., Ueno, M., \& Koyama, K. 2000, PASJ, 52, L73 